\title{
Lean mass, not fat mass, is associated with lung function in male and female children with asthma
}

\author{
Megan E. Jensen ${ }^{1,2}$, Peter G. Gibson ${ }^{1,2}$, Clare E. Collins ${ }^{3}$ and Lisa G. Wood ${ }^{1,2}$
}

BACKGROUND: Whether body composition is associated with lung function in asthmatic children has not been investigated. This study aimed to primarily investigate whether BMI $z$-score and body composition were associated with respiratory function in asthmatic children.

METHODS: In a cross-sectional study, male ( $n=27$; mean age: 11.9 y (SD: 2.3$)$ ) and female ( $n=21$; mean age: 13.6 y (SD: 2.2)) asthmatic children underwent clinical assessment.

RESULTS: BMI $z$-score was associated with forced expiratory volume in $1 \mathrm{~s}\left(\mathrm{FEV}_{1} ; r=0.458\right)$, forced vital capacity (FVC; $r=$ $0.477)$, and total lung capacity (TLC; $r=0.451)$ in males only $(P<$ 0.05). Total lean mass was associated with FEV $(r=0.655)$, FVC $(r=0.562)$, and TLC $(r=0.635)$ in males, as was thoracic lean mass (FEV $(r=0.573)$, FVC $(r=0.526)$, and TLC $(r=0.497) ; P<$ 0.05). TLC was associated with total $(r=0.522)$ and thoracic $(r=$ $0.532)$ lean mass in females $(P<0.05)$. Fat mass was not associated with lung function in this group.

CONCLUSION: Lean mass, not fat mass, is associated with lung function in children with asthma. The positive association between BMI $z$-score and respiratory function in male children is driven by lean mass. Although body weight can be easily monitored in the clinical setting, body composition can provide important information. Future research exploring lean mass and lung function associations could inform future interventions.

O besity is a highly and increasingly prevalent condition in children (1), with approximately one in four Australian children and one in three American children now above the 85 th BMI percentile $(2,3)$. Importantly, obesity is more prevalent in children with asthma than the general population, and obesity is a clinically significant risk factor for asthma in children and adults $(4,5)$. The etiology of the link between obesity and asthma is unknown, and proposed mechanisms include a proinflammatory effect of obesity and a physiological effect resulting from body composition changes associated with obesity $(4,6)$. Additionally, there appear to be gender-specific differences in the mechanisms (7).

Evidence suggests that obesity alters respiratory function in adults, with and without asthma. Reductions in forced expiratory volume in $1 \mathrm{~s}\left(\mathrm{FEV}_{1}\right)$ and forced vital capacity (FVC) have been reported in obese adults compared to healthy controls (8). However, obesity in adults appears to have a more clinically important detriment on static lung function (6), with a strong inverse relationship between BMI and both functional residual capacity and expiratory reserve volume reported $(6,9,10)$. In children, the data are inconsistent. Spirometry values appear largely unaffected or even slightly increased in overweight and obese children compared to healthy weight controls (11-13). Recent publications have indicated that static lung functions (residual volume, functional residual capacity, and expiratory reserve volume) are reduced in overweight and obese children similar to adults $(11,13,14)$.

Differentiating fat vs. lean mass may be an important factor in examining the relationship between lung function and obesity. Previous studies have indicated that the regional distribution of body composition may be important to respiratory function in asthmatic and nonasthmatic adults (15-17) and nonasthmatic children (18). In adults, total and regional fat mass have been negatively associated with dynamic and static lung function, with more evident differences in associations between males and females $(16,17)$. Increases in dual-energy $\mathrm{x}$-ray absorptiometry (DEXA)-measured thoracic adiposity in adults, with and without asthma, have been associated with poorer respiratory function $(16,17)$, indicating that this region may be an important anthropometric measure. However, its use and reproducibility have not been reported in children. Only one study has assessed the association between DEXAmeasured percentage body fat and lung volumes in children (18). This study was conducted in morbidly obese children without asthma and reported reductions in static lung function with increased adiposity (18). However, the relationship between body composition and lung function in male and female children with asthma has not been described.

We hypothesize that body composition is associated with lung function in asthmatic children and adolescents, particularly in the thoracic region. Therefore, the primary aim of this study was to investigate whether indirect (BMI $z$-score) and direct (DEXA) measures of body composition are associated with lung function in school-aged children with asthma,

'Department of Respiratory and Sleep Medicine, Hunter Medical Research Institute, New South Wales, Australia; ${ }^{2}$ Centre for Asthma and Respiratory Diseases, University of Newcastle, New South Wales, Australia; ${ }^{3}$ Faculty of Health, School of Health Sciences, University of Newcastle, New South Wales, Australia. Correspondence: Lisa G. Wood (lisa.wood@newcastle.edu.au) 
using partial correlations. The secondary aim was to assess the reproducibility of DEXA-measured total and regional body composition in children, specifically in the thoracic region, by examining agreement between dual total body scans using intraclass correlation coefficients.

\section{RESULTS}

Subject characteristics are summarized by gender as shown in Table 1. All participants were Caucasian. Females were significantly older but of similar height, weight, and BMI $z$-score to males. As expected, the DEXA-measured mean percentage body fat (in the thoracic region) was significantly greater in females than that in males (Table 1). Lung function, medication use, and airway reactivity were similar between males and females. However, males had a significantly poorer Juniper asthma control questionnaire score (Table 1$)$. There was also a nonstatistically significant difference $(P=0.134)$ in the proportion of males vs. females classified as having intermittent $(24.0$ vs. $47.6 \%$ ), mild ( 24.0 vs. $33.3 \%$ ), moderate ( 36.0 vs. $14.3 \%$ ), and severe (16.0 vs. $4.8 \%$ ) asthma. Urinary cotinine levels were negligible in all the participants, and therefore, tobacco exposure was not given further consideration.

In males, there was a significant positive correlation between BMI $z$-score and FVC (liters), $\mathrm{FEV}_{1}$ (liters), and total lung capacity (TLC) (liters) (Table 2), whereas no associations between BMI $z$-score and lung function/volumes reached statistical significance in females (Table 3 ). There were moderately strong positive associations for both total and thoracic lean mass with $\mathrm{FEV}_{1}$ (liters), FVC (liters), and TLC (liters) in males (Table 2). In females, the associations between total and thoracic lean mass and TLC (liters) reached statistical significance (Table 3). No statistically significant associations for either total or thoracic fat mass with respiratory function variables were detected in males (Table 2) or females (Table 3). Strong correlations were observed between the first and second DEXA scan for the total and thoracic lean and fat mass (Table 4).

\section{DISCUSSION}

To our knowledge, this is the first study to investigate whether standardized BMI and DEXA-measured body composition are associated with dynamic and static lung function in schoolaged children with asthma. Different associations between body composition and lung function were detected for male and female children, with a greater number of associations noted for male children. Moderately strong positive associations between BMI $z$-score and $\mathrm{FEV}_{1}, \mathrm{FVC}$, and TLC in male children appeared to be driven by lean mass, as indicated by the positive association between lean mass (total and thoracic) and $\mathrm{FEV}_{1}, \mathrm{FVC}$, and TLC. In females, the association between total

Table 1. Characteristics of male and female children with asthma

\begin{tabular}{|c|c|c|c|}
\hline Subject characteristics & Asthmatic females $(n=21)$ & Asthmatic males ( $n=27)$ & $P$ value \\
\hline Age $(y) ;$ mean \pm SD & $13.6 \pm 2.2$ & $11.9 \pm 2.3^{\mathrm{a}}$ & 0.014 \\
\hline Weight $(\mathrm{kg}) ;$ mean $\pm \mathrm{SD}$ & $69.7 \pm 21.3$ & $67 . \pm 25.3$ & 0.746 \\
\hline Height $(\mathrm{cm})$; mean \pm SD & $158.9 \pm 7.9$ & $157.4 \pm 14.1$ & 0.672 \\
\hline BMI z-score (SDS); median (IQR) & $1.8(1.3,2.1)$ & $2.0(1.7,2.4)$ & 0.107 \\
\hline Atopic (Y/N); $n(\%)$ & $15 / 6(71.4 \%)$ & $22 / 5(81.5 \%)$ & 0.498 \\
\hline Airway hyperresponsiveness (Y/N); $n(\%)$ & $15 / 5(75 \%)$ & $15 / 9(62.5 \%)$ & 0.711 \\
\hline Asthma control questionnaire & $0.4(0.4,0.7)$ & $1.0(0.4,1.4)^{\mathrm{a}}$ & 0.047 \\
\hline $\mathrm{PD}_{15}(\mathrm{ml}) ;$ median $(\mathrm{IQR})$ & $2.3(0.5,8.5)$ & $3.7(1.4,9.2)$ & 0.395 \\
\hline Dose response slope (\%/ml); median (IQR) & $1.9(0.7,15.3)$ & $2.09(0.52,5.09)$ & 0.367 \\
\hline $\mathrm{ICS}(\mathrm{Y} / \mathrm{N}) ; n(\%)$ & $9 / 12(42.9 \%)$ & $10 / 17(37.0 \%)$ & 0.455 \\
\hline Beclomethasone equivalents (beqs); median (IQR) & $233(100,400)$ & $200(125,400)$ & 0.866 \\
\hline $\mathrm{FEV}_{1} \%$ predicted $(\%) ;$ mean $\pm \mathrm{SD}$ & $90.6 \pm 9.3$ & $91.4 \pm 12.6$ & 0.811 \\
\hline FVC $\%$ predicted (\%); mean \pm SD & $98.0 \pm 9.0$ & $100.3 \pm 11.4$ & 0.453 \\
\hline $\mathrm{FEV}_{1} / \mathrm{FVC}(\%) ;$ mean $\pm \mathrm{SD}^{\mathrm{a}}$ & $81.0 \pm 6.5$ & $77.7 \pm 6.4$ & 0.082 \\
\hline TLC (liters); median (IQR) & $4.2(3.7,4.7)$ & $4.0(3.4,5.0)$ & 0.951 \\
\hline Functional residual capacity (liters); median (IQR) & $2.2(1.5,2.5)$ & $1.9(1.6,2.0)$ & 0.509 \\
\hline Expiratory reserve volume (liters); median (IQR) & $0.8(0.6,1.3)$ & $0.9(0.7,1.1)$ & 0.893 \\
\hline Residual volume (liters); median (IQR) & $1.0(0.7,1.4)$ & $0.9(0.8,1.1)$ & 0.557 \\
\hline Total body fat (\%); mean \pm SD & $44.0 \pm 10.0$ & $37.6 \pm 12.0$ & 0.057 \\
\hline Android fat (\%); mean \pm SD & $50.5 \pm 7.8$ & $45.2 \pm 10.9$ & 0.079 \\
\hline Gynoid fat (\%); mean \pm SD & $49.8 \pm 11.3$ & $43.1 \pm 14.5$ & 0.106 \\
\hline Thoracic fat (\%); mean \pm SD & $39.9 \pm 13.4$ & $31.1 \pm 13.2^{\mathrm{a}}$ & 0.038 \\
\hline
\end{tabular}

asignificantly different between asthmatic female and asthmatic male group, $P<0.05$.

$\mathrm{IQR}$, interquartile range; SDS, SD score. 
Table 2. Partial correlations between BMI z-score, total fat mass, and total lean mass measurements and respiratory function in males with asthma

\begin{tabular}{|c|c|c|c|c|c|}
\hline \multirow[b]{3}{*}{ Lung function variables } & \multicolumn{5}{|c|}{ Asthmatic males ( $n=27)$ : body composition assessment } \\
\hline & \multirow[b]{2}{*}{ BMI z-score (SDS) } & \multicolumn{2}{|c|}{ Fat mass (kg) } & \multicolumn{2}{|c|}{ Lean mass $(\mathrm{kg})$} \\
\hline & & Total body & Thoracic & Total body & Thoracic \\
\hline FVC (liters) & $0.477^{*}$ & 0.204 & 0.141 & $0.562^{*}$ & $0.526^{*}$ \\
\hline $\mathrm{FEV}_{1} / \mathrm{FVC}(\%)$ & 0.122 & -0.065 & -0.114 & 0.391 & 0.265 \\
\hline Functional residual capacity (liters) & -0.038 & -0.016 & 0.069 & 0.150 & 0.084 \\
\hline Expiratory reserve volume (liters) & -0.211 & -0.377 & -0.380 & -0.246 & -0.331 \\
\hline Residual volume (liters) & 0.118 & 0.261 & 0.347 & 0.329 & 0.325 \\
\hline
\end{tabular}

Data presented as partial correlations adjusted for age and height.

FEV, forced expiratory volume; FVC, forced vital capacity; SDS, SD score; TLC, total lung capacity.

$* P<0.05$.

Table 3. Partial correlations between BMI z-score, total fat mass, and total lean mass measurements and respiratory function in females with asthma

\begin{tabular}{|c|c|c|c|c|c|}
\hline \multirow[b]{3}{*}{ Lung function variables } & \multicolumn{5}{|c|}{ Asthmatic females ( $n=21)$ : body composition assessment } \\
\hline & \multirow[b]{2}{*}{ BMI z-score (SDS) } & \multicolumn{2}{|c|}{ Fat mass (kg) } & \multicolumn{2}{|c|}{ Lean mass (kg) } \\
\hline & & Total body & Thoracic & Total body & Thoracic \\
\hline FVC (liters) & 0.315 & 0.299 & 0.343 & 0.378 & 0.346 \\
\hline $\mathrm{FEV}_{1} / \mathrm{FVC}(\%)$ & 0.186 & -0.159 & -0.193 & -0.368 & -0.470 \\
\hline Functional residual capacity (liters) & -0.188 & -0.147 & -0.106 & -0.039 & -0.043 \\
\hline Expiratory reserve volume (liters) & -0.216 & -0.213 & -0.120 & -0.406 & -0.495 \\
\hline Residual volume (liters) & -0.202 & -0.133 & -0.193 & 0.272 & 0.346 \\
\hline
\end{tabular}

Data presented as partial correlations adjusted for age and height.

FEV, forced expiratory volume; FVC, forced vital capacity; SDS, SD score; TLC, total lung capacity.

$* P<0.05$.

lean mass and thoracic lean mass with TLC reached statistical significance, but no associations were noted for BMI $z$-score. Furthermore, no associations were observed between lung function and fat mass in either male or female children with asthma. Our data suggest that lung function measures $\left(\mathrm{FEV}_{1}\right.$, FVC, and TLC) in children may not be impaired by total body weight and, in fact, is positively influenced by increases in lean mass only. This has not been previously reported in children with asthma using an objective measure of body composition.

A positive association between lung function and BMI $z$-score was observed in males, whereas there was no significant association for females. However, the associations between total and thoracic lean mass with $\mathrm{FEV}_{1}, \mathrm{FVC}$, and TLC indicate that lean mass is driving the association between BMI $z$-score and lung function in this group of male children. In addition, total and thoracic lean mass were associated with TLC in females. Recent data have suggested that higher childhood BMI is associated with higher adult $\mathrm{FEV}_{1}$ and FVC (19). However, these seemingly positive effects disappeared after adjusting for indirect measures of lean body mass (handgrip
Table 4. Intraclass correlations between dual DEXA scans

\begin{tabular}{lcc}
\hline $\begin{array}{l}\text { DEXA manually adjusted } \\
\text { region }(n=48)\end{array}$ & $\begin{array}{c}\text { Intraclass correlation } \\
\text { coefficients }\end{array}$ & $95 \% \mathrm{Cl}$ \\
\hline Fat mass & 0.997 & $(0.995,0.999)$ \\
Total body fat mass $(\mathrm{kg})$ & 0.974 & $(0.956,0.992)$ \\
Thoracic fat mass $(\mathrm{kg})$ & & \\
Lean mass & 0.991 & $(0.985,0.997)$ \\
Total body lean mass $(\mathrm{kg})$ & 0.943 & $(0.905,0.981)$ \\
\hline Thoracic lean mass $(\mathrm{kg})$ & &
\end{tabular}

$\mathrm{Cl}$, confidence interval; DEXA, dual-energy $\mathrm{x}$-ray absorptiometry.

strength and skin-fold test derivations). An increased BMI indicates not only increased fat mass but also increased lean mass due to the effects of overnutrition and weight bearing. This highlights the importance of assessing body composition, as well as BMI, when interpreting the associations between body weight and lung function. An increase in lean mass may represent increased strength of the diaphragm and chest wall 


\section{Articles $\mid$ Jensen et al.}

to expand and contract with ventilation producing a greater $\mathrm{FEV}_{1}, \mathrm{FVC}$, and subsequently TLC. It may be hypothesized that exercise interventions targeting this region (e.g., resistance training) are needed to evaluate whether increases in lean mass, particularly thoracic lean mass, can be achieved in children with asthma, and subsequently whether this is associated with improvements in lung function.

Given that muscle accretion is greater in males than that in females, particularly during puberty (20), this may explain why fewer associations were observed between lean mass and respiratory function in female children. During puberty, males accumulate a higher amount of lean mass, at a much quicker rate than females and over a longer period $(20,21)$. Gender differences have also been noted in adults. Recent studies in asthmatic and nonasthmatic adults have reported similar positive associations between lean mass and respiratory function in males only $(16,17)$, whereas negative associations between lean mass and lung function have been reported in female adults with asthma (17).

Furthermore, the current study did not detect significant associations between fat mass and lung function in this group of male and female children with asthma. This is in contrast to the negative associations between fat mass and lung function detected in adults with and without asthma $(16,17)$. A recent study of overweight and obese adults with asthma reported an inverse association between dynamic and static lung function and fat mass in females using partial correlation analysis (17). Conversely, in males, lung function was not in association with fat mass (17). The current study results also contrast with those from a study in nonasthmatic obese children, which found a significant negative association between DEXA-measured subtotal and trunk \%body fat and TLC \%predicted, functional residual capacity \%predicted, and residual volume \%predicted (18). However, this population sample was morbidly obese, and authors did not separate the analysis by gender (18). Of note in the current study is the trend toward a moderate negative association between static lung function and fat mass in both male and female children. Although our data suggest that in children and adolescents with asthma, lean mass may be more important than fat mass in relation to respiratory function, studies powered to detect significant relationships between respiratory function and body composition are needed to confirm these findings. It is possible that given a large sample size and a more adequately powered study, the nonsignificant negative correlations between lung function and fat mass that were observed in the present study may become significant. Therefore, follow-up studies are needed. Nonetheless, the associations between lean mass and lung function are more important in this population, because these were significant despite the small sample size.

As a secondary aim, the agreement between DEXA scans for the thoracic region was assessed. The DEXA-measured thoracic region has been used previously in adult studies $(16,17)$, but its reproducibility and use in children have not been reported. This is the first study to indicate that the manually determined thoracic region body composition by
DEXA assessment appears to be a reproducible measurement in school-aged children with asthma. Given the associations observed between thoracic lean mass and lung function, assessment of this region may be relevant in future research studies that include respiratory outcomes as a primary end point. Therefore, future studies that assess the reliability and validity of this regional measurement are warranted.

This study presents novel information regarding lung function and body composition in male and female children with asthma. It is acknowledged that the capabilities of this study were limited by sample size. The strength of the correlation factor $(r)$ may have been influenced by the small sample size. This was the secondary analysis of the data and was not powered to detect significant associations between lung function and body composition. The results serve as an exploratory analysis to inform future studies. In addition, the uneven demographics between males and females (asthma control questionnaire, age) are the limitations of this study. Females had better asthma control, and this may have influenced the associations between lung function and body composition detected. Stratification by asthma control/severity could be considered in future studies. The significantly higher \%thoracic fat mass in females was expected, as this is likely attributable to breast tissue development in females. We expect that these differences in regional body composition may have contributed to the differences that we observed. This reaffirms the importance in assessing males and females separately when examining body composition. Although the analyses were adjusted for age, future research may benefit from separating prepubertal and pubertal participants to better control for the influence of puberty on body composition. Finally, this study did not include a nonasthmatic control group. Our objective was to explore associations in asthmatic children, given the emerging evidence of the obese asthma phenotype in children. Future research may investigate if such associations exist in nonasthmatic children.

Agreement between dual DEXA scans was strong in this group of children. However, it is acknowledged that further research is needed to investigate the reliability, validity, and clinical importance of the DEXA-measured thoracic region. In addition, this study did not explore the relationship between body composition and lung function in younger children. The use and reproducibility of DEXA may be reduced in younger children, due to increased risk of movement during the test, and therefore, the results cannot be extrapolated to children under $8 \mathrm{y}$ of age. The general application of DEXA in the clinical setting is likely limited in terms of access and cost (purchase, maintenance, software, and trained personnel required). It, therefore, is of interest to compare the use of alternative body composition assessment methods, which may be more cost effective for general practice.

While monitoring body weight is a simple and practical way of tracking weight change in the clinical setting, our data demonstrate that body composition measurement can reveal important information about the relationship between body weight and lung function in children and should be considered in future research studies. In this group of school-aged children with asthma, the 


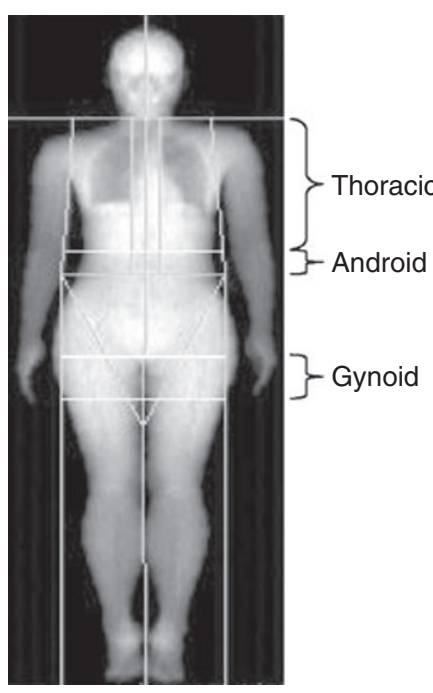

Figure 1. Dual-energy $x$-ray absorptiometry scan depicting key regions of interest. Image prior to manual adjustment.

relationship between BMI $z$-score and $\mathrm{FEV}_{1}, \mathrm{FVC}$, and TLC in males appears to be driven by lean mass. Positive associations between total and thoracic lean mass and TLC were also detected in female children. It may be hypothesized that improvements in total lean mass, including thoracic lean mass, could potentially yield improvements in respiratory function for children with asthma, detectable through routine spirometry assessment, and potentially warrants testing in intervention studies. Furthermore, in contrast to adult literature, fat mass was not significantly associated with lung function in male or female children. Finally, results suggest that thoracic body composition measurement may be a reliable tool, and its clinical relevance warrants further evaluation. However, the practical implications and the clinical relevance of these findings, as well as the use and validity of the thoracic region measurement, require further exploration in a larger study powered to detect significant associations.

\section{METHODS}

Design

This is a secondary analysis of a cross-sectional study, published elsewhere (14). A subset of participants with asthma who completed lung plethysmography and dual DEXA scans were included in the current analysis.

\section{Study Participants}

Obese and nonobese participants aged 8-17 y, with asthma, were enrolled from the general community in Newcastle, Australia, using media advertisement, medical centers, John Hunter Hospital outpatient clinics, and school newsletters, during the period June 2009 to March 2011. Asthma was defined as a physician diagnosis and episodic respiratory symptoms in the past year. Participants were offered nutritional consult(s) with an accredited practicing dietitian (M.E.J.) and feedback on results in return for their participation in the study. Participants were reviewed by a pediatric respiratory physician. Exclusion criteria included metabolic or cardiac comorbidities, inflammatory disease or respiratory disorder other than asthma, or unexplained weight change during the past 3 mo. The study was approved by Hunter New England Human Research Ethics Committee and the University of Newcastle Human Research Ethics Committee (Newcastle, Australia). All participants gave written assent, and guardians gave written consent.

\section{Clinic Visits}

Participants attended the Hunter Medical Research Institute, John Hunter Hospital, for testing, and they were instructed to withhold antihistamines and asthma medications for $6-24 \mathrm{~h}$ prior to the visit, if applicable. Current asthma status was assessed using the Juniper asthma control questionnaire (22). Clinical asthma pattern was assessed using the Global Initiative for Asthma guidelines (23). Asthma stability was confirmed and defined as no exacerbation, respiratory tract infection, or oral corticosteroid use in the past $4 \mathrm{wk}$. Airway hyperresponsiveness to hypertonic saline (4.5\%) was defined by a $\geq 15 \%$ drop in baseline $\mathrm{FEV}_{1}$. Atopy was evaluated by a positive skin prick test to common allergen(s) (Aspergillus fumigatus, Alternaria tenius, dust mite (Dermatophagoides pteronyssinus), cockroach mix, grass mix). Tobacco smoke exposure was measured via urinary cotinine assay (NicAlert; Nymox Pharmaceutical, Hasbrouck Heights, NJ).

\section{Anthropometry}

Non-DEXA measurements. Weight and height were measured to nearest $100 \mathrm{~g}$ using $150 \mathrm{~kg}$ maximum scales (EB8271 NuWeigh; Newcastle Weighing Services, Newcastle, Australia) and 2 m wall-suspended measuring tape to nearest $0.1 \mathrm{~cm}$ with wall stop (Surgical and Medical Supplies Pty, Rose Park, Australia) with the children barefoot and wearing light clothing. BMI was calculated (weight $(\mathrm{kg}) / \mathrm{height}$ $\left.(\mathrm{m})^{2}\right)$ and converted to BMI $z$-scores (SD score (SDS)) (24).

DEXA measurements. Dual total body scans were conducted on all participants using DEXA (GE Lunar Prodigy; Medtel, Madison WI and GE Healthcare encore 2007 software version 11.40.004; GE Healthcare; Madison, WI). Participants were scanned on the appropriate setting as per the machine selection (thin/standard/thick mode). The participants arose from the table after the first scan, walked briefly around the room, and were then repositioned immediately by the same technician for the second scan. The scanner provided percentage total and regional body fat and absolute total and regional bone mineral density, fat mass, and lean mass. Manual adjustments were made to each scan to ensure the regions of the body that were contained within the set parameters. The height of the android region of interest was equal to $20 \%$ of the distance between the pelvis and the neck of femur, with the inferior border defined by the upper part of the pelvis. The height of the gynoid region was 1.5 times the height of the android region, with the upper border at the superior part of the trochanter major (Figure 1). The thoracic region was framed using the "scanner region of interest" function of the software. A box was first overlaid and then adjusted to ensure dissection through the acromioclavicular joint by two vertical lines bordering the rib cage, a horizontal line beneath the chin, and a horizontal line at the inferolateral limit of the rib cage. This region of interest has previously been described (16).

\section{Respiratory Function Assessment}

$\mathrm{FEV}_{1}$ and FVC were measured using spirometry (KoKo PD Instrumentation, Louisville, CO). For descriptive tabulation, spirometry values were expressed as a percentage of the predicted value (25). Lung volumes were measured using a plethysmograph (MedGraphics Elite Series Plethysmograph, St Paul, MN). The instruments were calibrated prior to patient assessment. The best of the three technically acceptable values was accepted for use in the primary statistical analysis.

\section{Statistical Analysis}

Subject characteristics are classified by gender and summarized in terms of counts $(n)$ and proportions (\%), means (with SD), and medians (with interquartile range). Partial correlations are reported by gender. Partial correlations, controlled for age and height, were used to investigate the association between BMI $z$-score, total body fat mass $(\mathrm{kg})$, and total body lean mass with respiratory variables. The average values for total fat and total lean mass $(\mathrm{kg})$ were calculated from the dual DEXA scans. To reduce any potential error from the assumption that the chosen reference values are applicable to our study group (26), lung function variables remained in their original scale (liters) for the partial correlations. Agreement between the DEXA scans was 
assessed using intraclass correlations. The intraclass correlation coefficient $(-95 \% \mathrm{CI},+95 \% \mathrm{CI})$ for each manually adjusted DEXA region of interest was reported. The data being pairs of measures, the estimator of the intraclass correlation coefficients was the Pearson correlation coefficient $(r)$. An $r>0.80$ indicates strong correlation between the two measurements. All tests were of size $\alpha=0.05$, and no adjustments were made for multiple comparisons. Intercooled Stata Version 11.0 for Windows (StataCorp, College Station, TX) was used for all statistical computations.

\section{ACKNOWLEDGMENTS}

The authors thank Majella Maher, Allyson Upward, and Jeff Pretto (John Hunter Hospital) and Joanne Smart and Amber Smith (University of Newcastle) for their assistance in data collection; the authors thank Patrick McElduff (University of Newcastle) for the extensive statistical consultation.

\section{STATEMENT OF FINANCIAL SUPPORT}

We acknowledge the financial support of University of Newcastle Priority Research Centre Research Higher Degree Scholarship, Australia (to M.E.J.); National Health and Medical Research Practitioner Fellowship, Australia (to P.G.G.); and National Health and Medical Research Council Career Development Fellowship, Australia (to C.E.C.).

Disclosure: The authors declare that they have no competing interests.

\section{REFERENCES}

1. Han JC, Lawlor DA, Kimm SY. Childhood obesity. Lancet 2010;375:173748.

2. Commonwealth Scientific Industrial Research Organisation (CSIRO) Preventative Health National Research Flagship \& The University of South Australia. 2007 Australian National Children's Nutrition and Physical Activity Survey Main Findings, 2008. (http://www.health.gov.au/internet/ main/publishing.nsf/Content/66596E8FC68FD1A3CA2574D50027DB86 /\$File/childrens-nut-phys-survey.pdf.).

3. Ogden CL, Carroll MD, Flegal KM. High body mass index for age among US children and adolescents, 2003-2006. JAMA 2008;299:2401-5.

4. Jensen ME, Collins CE, Gibson PG, Wood LG. The obesity phenotype in children with asthma. Paediatr Respir Rev 2011;12:152-9.

5. Beuther DA. Recent insight into obesity and asthma. Curr Opin Pulm Med 2010;16:64-70.

6. Salome CM, King GG, Berend N. Physiology of obesity and effects on lung function. J Appl Physiol 2010;108:206-11.

7. Scott HA, Gibson PG, Garg ML, Wood LG. Airway inflammation is augmented by obesity and fatty acids in asthma. Eur Respir J 2011;38:594-602.

8. Schachter LM, Salome CM, Peat JK, Woolcock AJ. Obesity is a risk for asthma and wheeze but not airway hyperresponsiveness. Thorax 2001;56:4-8.
9. Sin DD, Sutherland ER. Obesity and the lung: 4. Obesity and asthma. Tho$\operatorname{rax} 2008 ; 63: 1018-23$.

10. Jones RL, Nzekwu MM. The effects of body mass index on lung volumes. Chest 2006;130:827-33.

11. Rastogi D, Canfield SM, Andrade A, et al. Obesity-associated asthma in children: a distinct entity. Chest 2012;141:895-905.

12. Jensen ME, Wood LG, Gibson PG. Obesity and childhood asthma - mechanisms and manifestations. Curr Opin Allergy Clin Immunol 2012;12:18692.

13. Ruppel GL. What is the clinical value of lung volumes? Respir Care 2012;57:26-35; discussion 35-8.

14. Jensen ME, Gibson PG, Collins CE, Wood LG. Airway and systemic inflammation in obese children with asthma. Eur Respir J 2013;42:1012-9.

15. Sood A, Qualls C, Li R, et al.; CARDIA Investigators. Lean mass predicts asthma better than fat mass among females. Eur Respir J 2011;37:65-71.

16. Sutherland TJ, Goulding A, Grant AM, et al. The effect of adiposity measured by dual-energy X-ray absorptiometry on lung function. Eur Respir J 2008;32:85-91.

17. Scott HA, Gibson PG, Garg ML, et al. Relationship between body composition, inflammation and lung function in overweight and obese asthma. Respir Res 2012;13:10.

18. Li AM, Chan D, Wong E, Yin J, Nelson EA, Fok TF. The effects of obesity on pulmonary function. Arch Dis Child 2003;88:361-3.

19. Curry BA, Blizzard CL, Schmidt MD, Walters EH, Dwyer T, Venn AJ. Longitudinal associations of adiposity with adult lung function in the Childhood Determinants of Adult Health (CDAH) study. Obesity (Silver Spring) 2011;19:2069-75.

20. Veldhuis JD, Roemmich JN, Richmond EJ, et al. Endocrine control of body composition in infancy, childhood, and puberty. Endocr Rev 2005;26:11446.

21. Loomba-Albrecht LA, Styne DM. Effect of puberty on body composition. Curr Opin Endocrinol Diabetes Obes 2009;16:10-5.

22. Juniper EF, O’Byrne PM, Guyatt GH, Ferrie PJ, King DR. Development and validation of a questionnaire to measure asthma control. Eur Respir J 1999;14:902-7.

23. Global Initiative for Asthma. Pocket Guide for Asthma Management and Prevention in Children, 2005. (http://www.ginasthma.org/local/uploads/ files/GINAPocket05Clean_1.pdf. 10 Feb 2009.).

24. A SAS Program for the CDC Growth Charts. Centre for Disease Control and Prevention. (http://www.cdc.gov/nccdphp/dnpao/growthcharts/ resources/sas.htm.) Accessed 8 March 2012.

25. Hankinson JL, Odencrantz JR, Fedan KB. Spirometric reference values from a sample of the general U.S. population. Am J Respir Crit Care Med 1999;159:179-87.

26. Ruppel GL, Enright PL. Pulmonary function testing. Respir Care 2012;57:165-75. 